\title{
The Axon Protective Effects of Syringic Acid on Ischemia/ Reperfusion Injury in a Rat Sciatic Nerve Model
}

\author{
Mehmet TOKMAK ${ }^{1}$, Muserref Hilal SEHITOGLU², Yasemin YUKSEL ${ }^{3}$, Mustafa GUVEN ${ }^{4}$, Tarik AKMAN ${ }^{4}$ \\ Adem Bozkurt ARAS ${ }^{4}$, Umut YAKA ${ }^{1}$, Cengiz GOMLEKSIZ1 ${ }^{1}$, Serdar Baki ALBAYRAK ${ }^{1}$, Murat COSAR ${ }^{4}$ \\ ${ }^{1}$ Medipol University, Faculty of Medicine, Department of Neurosurgery, Istanbul, Turkey \\ ${ }^{2}$ Canakkale Onsekiz Mart University, Faculty of Medicine, Department of Medical Biochemistry, Canakkale, Turkey \\ ${ }^{3}$ Afyon Kocatepe University, Faculty ofMedicine, Department of Histology and Embryology, Afyon, Turkey \\ ${ }^{4}$ Canakkale Onsekiz Mart University, Faculty of Medicine, Department of Neurosurgery, Canakkale, Turkey
}

\section{ABSTRACT}

AIM: In the relevant literature, there is no experimental study that investigated the axon protective effects of syringic acid- a polyphenol compound- with an anti-oxidant capacity on ischemia/reperfusion injury.

MATERIAL and METHODS: The rats were randomly divided into four groups: Control group (no medication or surgical procedure), Sham group, Syringic acid group, and Methyprednisolone (MP) Group. Ischemia was achieved by abdominal aorta clamping and all animals were sacrificed 24 hours after ischemia. Harvested sciatic nerve segments were investigated histopathologically and for tissue biochemistry.

RESULTS: Ischemic fiber degeneration scores were found significantly lower in syringic acid and MP groups than sham group. Additionally, apoptosis-related cysteine peptidase caspase-3 immunostaining scores were lower in syringic acid and MP groups. Biochemically, superoxide dismutase and nuclear respiratory factor 1 values were significantly higher in syringic acid group compared to those of control and sham groups while malondialdehyde levels were significantly lower in the syringic acid group.

CONCLUSION: Syringic acid reduces oxidative stress and axonal degeneration in rat sciatic nerve after ischemia/reperfusion injury. Therefore, syringic acid may play a role in the treatment of peripheral nerve injuries due to ischemia/reperfusion.

KEYWORDS: Axon, Sciatic nerve, Syringic acid, Methylprednisolone, Reperfusion, Malondialdehyde, Nuclear respiratory factor 1, Superoxide dismutase

\section{INTRODUCTION}

I schemia is a reversible or irreversible condition due to hypoperfusion of systemic arterial blood to tissues and Lorgans. Ischemia disrupts cellular oxidative phosphorylation process and this in turn causes adenosine triphosphate (ATP)-dependent ionic pump failure and hyperinflux of calcium, sodium, and water into cells $(3,8)$. Meanwhile, adenine nucleotide breakdown is accelerated resulting in intracellular accumulation of hypoxantine which is the precursor of reactive oxygen derivatives (ROD) (4). Additionally, ischemia increases the synthesis of some proinflammatory gene products (leucocyte adhesion molecule, cytokines, etc.) and bioactive compounds (endothelin-1, thromboxane A2, etc.) in endothelial cells while it suppresses the expression and synthesis of some cyto-protective genes and their enzyme products including nitric oxide (NO) and prostacyclins (5). At this critical stage, reperfusion- the restoration of blood flow following ischemia- must be accomplished in order to prevent irreversible tissue/organ damage. It ihas been proven that the temporary occlusion of aorta may cause reperfusion injury in the sciatic nerves (17). 
We investigated the potential axon protective effects of syringic acid (SA)-a polyphenol compound, a derivative of benzoic acid- on sciatic nerve ischemia and reperfusion. We compared its axon-protective effects to those of methyprednisolone (MP) which was recently shown to be effective in axon protection in sciatic nerve injuries (27).

In this study, the potential beneficial effects of SA on rat sciatic nerves after ischemia/reperfusion were evaluated histopathologically and biochemically by measuring malonyldialdehyde (MDA), superoxide dismutase (SOD), nuclear respiratory factor-1 (NRF-1).

\section{MATERIAL and METHODS}

\section{Animals}

Prior to the start of the experiments, permission was obtained from Canakkale Onsekiz Mart University Animal Experiments Ethics Committee.

Twenty-four male Sprague-Dawley rats each weighing $300 \pm 25$ grams and 8 to 12 weeks old were used in the experiment. All rats were fed with standard pellet rat food (Bil-Yem Ltd, AnkaraTurkey) and water ad libitum. An automatic photoperiod with white fluorescent light was used to create an environment with 12 hours light/12 hour darkness. The temperature was set to $21 \pm 2{ }^{\circ} \mathrm{C}$ and humidity was set to $55-60 \%$. The methods used for animal experiments were organized in accordance with the protocols of the National Institute of Health Guide for the Care and Use of Laboratory Animals.

\section{Experimental Design}

Rats were randomly divided into four equal groups (consisting of six rats each):

Group 1: Control group ( $n=6$, no medication or surgical procedure)

Group 2: Sham group ( $n=6$, Single dose $1 \mathrm{ml} 10 \%$ ethanol was administered intraperitoneally at $5^{\text {th }}$ minutes following aorta occlusion. Rats were sacrificed at $24^{\text {th }}$ hours after ischemia)

Group 3: Syringic Acid Group-SA ( $\mathrm{n}=6$, Single dose $10 \mathrm{mg} /$ $\mathrm{kg}$, SA (Syringic Asid Sigma-Aldrich Interlab, Turkey) was administered intraperitoneally at $5^{\text {th }}$ minutes following aorta occlusion. Rats were sacrificed at $24^{\text {th }}$ hours after ischemia).

Group 4: Methylprednisolone Group-MP ( $n=6$, Single dose 30 $\mathrm{mg} / \mathrm{kg}$ MP (Prednol, Mustafa Nevzat, Turkey) was administered intraperitoneally immediately following spinal cord ischemia (SCl).

\section{Dosage}

The dosage was determined as $10 \mathrm{mg} / \mathrm{kg}$ body weight based on preliminary studies with various doses $(50,75,100 \mathrm{mg})$ to reveal the biological effects of SA.

\section{Reagents}

Syringic acid ( $\geq 98 \%$ HPLC) was obtained from Sigma-Aldrich (St Louis, MO, USA). MP was obtained from Mustafa Nevzat Drug Industry Inc. (Istanbul, Turkey). The drugs were dispersed with isotonic $\mathrm{NaCl} 0.9 \%$. The protein concentrations were indicated by the Bradford method using Bradford reagent (Catalogue No. B6916-1KT) (Sigma Aldrich, St Louis, MO, USA). SOD Assay Kit (Catalogue No. 19160) was obtained from Sigma-Aldrich (St Louis, MO, USA). Lipid peroxidation (MDA) assay kit (Cat. No. MAK085) was obtained from SigmaAldrich (St.Louis, MO, USA). Rat Nuclear Respiratory Factor 1 ELISA Kit (Cat. No. CK-E90555) was obtained from Hangzhou Eastbiopharm Co. Ltd. (Hangzhou, China). We preferred ethanol in sham group instead of serum physiologic since syringic acid could be solved in ethanol.

\section{Surgical Procedure}

Rats were administered premedication; intraperitoneal ketamine $(50 \mathrm{mg} / \mathrm{kg}$ ) and xylazine $(5 \mathrm{mg} / \mathrm{kg})$. Anesthesia was continued with ketamine injections at intervals without intubation or mechanical ventilation. Surgical approach was supine position. After the operating field was prepared in a sterile fashion, laparotomy was performed with a standard midline incision. After retracting the intestines laterally, the retroperitoneum was opened and the abdominal aorta reached. Aorta occlusion was induced by cross-clamping the aorta with mini aneurysm clip between just below the left renal artery and just proximal to the aortic bifurcation. Loss of aortic pulse was confirmed by palpation. The duration of ischemia was set at 45 minutes and later the cross clamps were removed and distal reperfusion was observed visually. At the end of the procedure, the abdominal wall was closed with $5 / 0$ prolene sutures. Animals in control group underwent a surgical procedure similar to the other groups but the aorta was not clamped. Animals were fed a standard diet and water ad libitum in their cages after surgery. At $24^{\text {th }}$ hour all animals were anesthetized with penthobarbital $(20 \mathrm{mg} / \mathrm{kg})$ and sacrificed. Sciatic nerves were removed full length via the gluteal approach in all animals. Half of the specimen taken for histopathological investigation and it was fixed in formalin for 7 days. The other half was stored in a freezer at $-80{ }^{\circ} \mathrm{C}$ for biochemical estimations.

\section{Histopathological Evaluation}

The sciatic nerve segments were fixed in 10\% neutral formalin and then each sample cut into two pieces. One piece was used for transverse and the other is for longitudinal section. After processed histologically, tissue samples were embedded in parafin and $5 \mu \mathrm{m}$ in thickness sections were taken. Tissue samples were stained with hematoxylin-eosin (H-E) (Sigma) and Modified Gomori trichrome solution (Bio Optica, Milano, Italy) (according to the manufacturer's protocol) in order to evaluate the neuronal damage and myelination status. Sciatic nerves were graded for ischemic fiber degeneration (IFD) using previously described method. The fibers were evaluated for IFD according to axonal changes such as swollen or shrunken axons and light or dark appearance. Myelin changes were examined in terms of breakdown or collapse. The sections were graded from 0 to 4 for IFD based on the percentage of IFD as follows: $\leq 2 \%, 3-25 \%, 26-50 \%, 51-75 \%$, and $>75 \%$, respectively (Table I). Each section of sciatic nerve was examined under light microscope. 
Table I: Ischemic Fiber Degeneration (IFD) Scores of Groups According to Mitsui et al. (17)

\begin{tabular}{ccccc}
\hline Rats & \multicolumn{5}{c}{ Scores of the groups for IFD } \\
\hline $\mathbf{1}$ & Control & Ischemia & Syringic acid & MP \\
\hline $\mathbf{2}$ & 0 & 3 & 2 & 1 \\
\hline $\mathbf{3}$ & 0 & 2 & 1 & 1 \\
\hline $\mathbf{4}$ & 0 & 2 & 2 & 1 \\
\hline $\mathbf{5}$ & 0 & 3 & 2 & 1 \\
\hline $\mathbf{6}$ & 0 & 2 & 1 & 1 \\
\hline $\mathbf{7}$ & 0 & 2 & 1 & 2 \\
\hline $\mathbf{8}$ & 0 & 3 & 2 & 1 \\
\hline Mean value & 0 & 2 & 1 & 1 \\
\hline
\end{tabular}

\section{Caspase-3 Immunohistochemistry Protocol}

The expressions of caspase-3 was investigated by immunohistochemistry. To summarize the protocol; following dewaxing, washing and rehydration of the slides, microwave heating in citrate buffer was used for antigen retrieval. Endogenous peroxidase was blocked by $3 \%$ hydrogen peroxidase in methanol. Then, sections were incubated with caspase-3 primary antibody (dilution 1:100)(AB3623, Millipore, California) at $4^{\circ} \mathrm{C}$ overnight. The next day, Horseradish peroxidase (HRP) secondary antibody kit (Anti-polyvalent HRP, Lab Vision Corp, Fremont, CA) was applied as a secondary antibody according to the manufacturer' protocol. 3-amino-9-ethylcarbazole (AEC) kit (Lab Vision Corp, Fremont, CA) was used for chromogen and then the sections were counterstained with Mayers hematoxylin and mounted with water based mounting medium. For evaluating the percentage of caspase-3 positive cells, fibers were scored using previously described method from 0 to 4 as follows: $<5 \%, 6-15 \%, 16-25 \%, 26-35 \%$ and $>35 \%$, respectively. All the sections were observed under a microscope (Eclipse E-600 Nikon, Japan). Image Analysis Software (NIS Elements Nikon, Japan) was used for assessing the samples.

\section{Statistical Analysis}

All experimental data were analyzed by SPSS for Windows 18 statistical analysis software. The Kruskal-Wallis test was used to analyze the differences between the groups and MannWhitney $U$ test was performed for pairwise comparisons. $p<0.05$ value was considered to be statistically significant.

\section{Biochemical Investigations of Sciatic Nerve Tissues}

After macroscopic analysis, rat tissues were kept at $-80^{\circ} \mathrm{C}$. For biochemical investigation, MDA, NRF1 levels and SOD activities from each supernatant were measured in duplicate with highly sensitive enzyme linked immunosorbent assay (ELISA) spectrophotometry, respectively. The protein concentrations were indicated by the Bradford method using Bradford reagent (Sigma Aldrich, Bradford reagent-B6916-1KT, USA). All the data was defined as the mean \pm standard deviation (SD) results based on per mg of protein.

\section{Nuclear Respiratory Factor-1 (NRF1)}

Rat NRF1 ELISA kit (Hangzhou Eastbiopharm CO., LTD., China) was used to assay NRF1 on the basis of the Biotin double antibody sandwich technology. Absorbances of each well were measured under $450 \mathrm{~nm}$ wavelength. The results were expressed as $\mathrm{ng} / \mathrm{ml}$ NRF1 per milligram protein $\left(\mathrm{ng} \cdot \mathrm{ml}^{-1}\right.$. mg protein ${ }^{-1}$ ).

\section{Superoxide Dismutase (SOD)}

Tissue SOD activity was measured with SOD assay kit (SigmaAldrich-19160-St.Louis, MO, USA) using highly sensitive ELISA spectrophotometry. The IC50 (50\% inhibition activity of SOD) values was determined by this colometric method under $450 \mathrm{~nm}$. The results were expressed as U/ml SOD per milligram protein (U. $\mathrm{ml}^{-1} . \mathrm{mg}$ protein $\left.{ }^{-1}\right)$.

\section{Lipid Peroxidation (MDA)}

Lipid peroxidation is determined by the reaction of MDA with thiobarbituric acid (TBA) to form a colorimetric (532 nm) product, proportional to the MDA present. Lipid peroxidation (MDA) assay kit (Sigma-Aldrich-MAK085- St.Louis, MO, USA) was used for determining MDA levels. The results were expressed as nmole/ml MDA per milligram protein (nmole. $\mathrm{ml}^{-1}$. mg protein $\left.{ }^{-1}\right)$.

\section{Statistical Analysis}

Results were subjected to one-way analysis of variance (ANOVA) using SPSS 21.0 software (SPSS Inc., USA). Differences among the groups were obtained using Tukey's test option. Statistical significance was accepted as $p<0.05$. All data was expressed as mean \pm standard deviation (SD) in each group.

\section{RESULTS}

\section{Histopathological Results}

Ischemic Fiber Degeneration: For histopathological examination of rat sciatic nerves, $\mathrm{H}-\mathrm{E}$ and Gomori trichrome staining were performed. A nerve fiber consists of an axon and a myelin sheath formed by Schwann cells. Myelin does not stain well in a typical $\mathrm{H}-\mathrm{E}$ preparation because of its high lipid 
content that is lost in the staining process. Neurokeratin content can be seen in this area, a sparse proteinaceous network that is the remnants of the myelin sheath following removal of fatty material. $\mathrm{H}-\mathrm{E}$ staining demonstrates centrally located nerve fiber, which is acidophilic, and surrounding pale-staining myelin sheath. After stained with Gomori trichrome solution, endoneurium was stained as green and neurokeratin of myelin was in red and nucleus of Schwann was in blue colour. In longitudinal sections, the myelin sheath was observed in elongated columns. In transverse sections, centrally placed axons, ensheathing Schwann cells together with endoneurium were observed.

In the control rats, myelinated area appeared in ordered structure showing neither axonal shrunken nor its swollen
(Figures 1A, 2A). Ischemic fiber degeneration (IFD) was graded based on the previous study of Mitsui et al. (17). There was some ischemic fiber degeneration in ischemia group (Figures 1B, 2B). Axonal swelling and shrinkage were observed. The average myelin loss and disorganisation were significantly more in this group in comparison to the controls $(p<0.05)$ and this deterioration was partly restored by syringic acid and MP group. Treatment with syringic acid significantly improved morphology of axon-myelination and it decreased ischemic fiber degeneration (Table I, Figures 1C, 2C) $(p<0.05)$. MP also significantly reduced ischemic fiber degeneration (Figures 1D, $2 D)(p<0.05)$. However, restoration of the nerve fibers was more prominent by treatment with MP group compared to the syringic acid group.

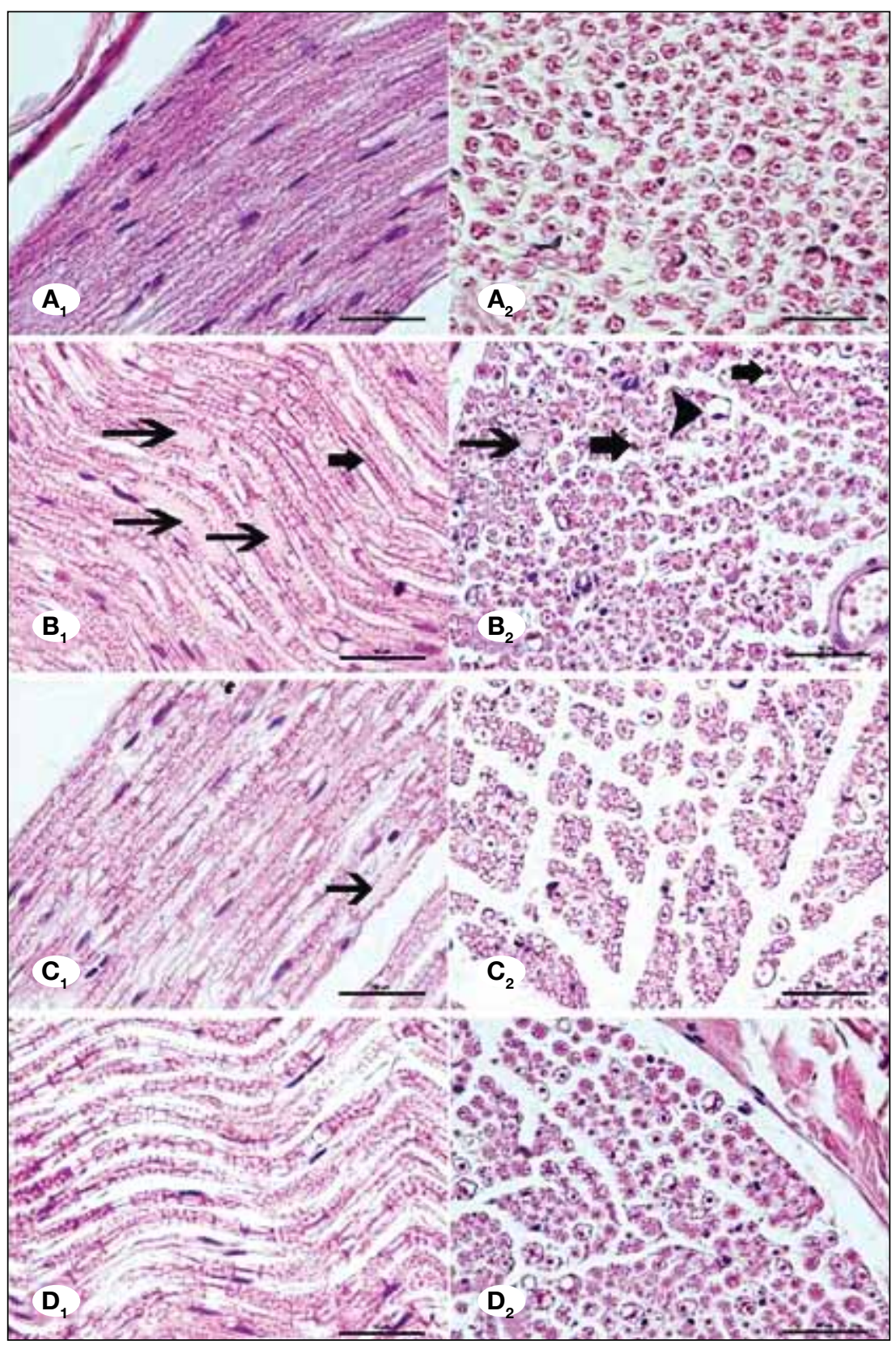

Figure 1: Control group showing normal morphology (A1-A2). Ischemia group showing axonal degeneration (arrows) (B1) swollen nerve fiber with myelin loss (arrowhead) and shrunken and darky axons (thick arrows) (B2). Syringic acid group showing less axonal and myelin degeneration than ischemia group (C1C2).MP group showing less axonal and myelin degeneration than syringic acid group (D1-D2). (Photomicrographs of longitudinal (A1,B1,C1,D1) and transverse $(A 2, B 2, C 2, D 2)$ sections from the sciatic nerves $(H-E, X 400$, scale bar $=50 \mu \mathrm{m})$. 
Tokmak M. et al: The Axon Protective Effects of Syringic Acid

Caspase-3 Expression of Sciatic Nerve Fibers: Caspase-3, an effector caspase, is activated in both intrinsic and the extrinsic pathway of apoptosis. Caspase-3 protein expression were observed by immunohistochemistry and graded based on the scoring system of Schmeichel et al. (22). Stained sections were examined under x40 magnification. Figures show the transvers sections from the nerve fibers under $\times 100$ magnification with immersion oil. Caspase-3 staining were observed in both axonal and myelinated region. The caspase expression of the control group was negative (Figure $3 \mathrm{~A}$ ). The percentage of positive nerve fibers for caspase- 3 in ischemic nerves was significantly higher than in controls (Table II, Figure $3 \mathrm{~B}, \mathrm{p}<0.05)$. Less positive staining was found in the syringic acid (Figure 3C) and MP (Figure 3D) groups. There was no significant difference in caspase-3 expression between syringic acid and MP groups ( $p>0.05)$.

In summary, peripheral nerves subjected to ischemia reperfusion injury underwent IFD with a breakdown in the axon and myelin sheath and apoptosis was increased. Treatment with syringic acid and MP reduced IFD and excess apoptosis.

\section{Biochemical Results}

The mean and standard deviation values of NRF1, SOD and MDA in each group were given in Table III and Figure 4A-C. NRF1 levels of ischemia/reperfusion group were found to be lower compared to the other groups and this was statistically

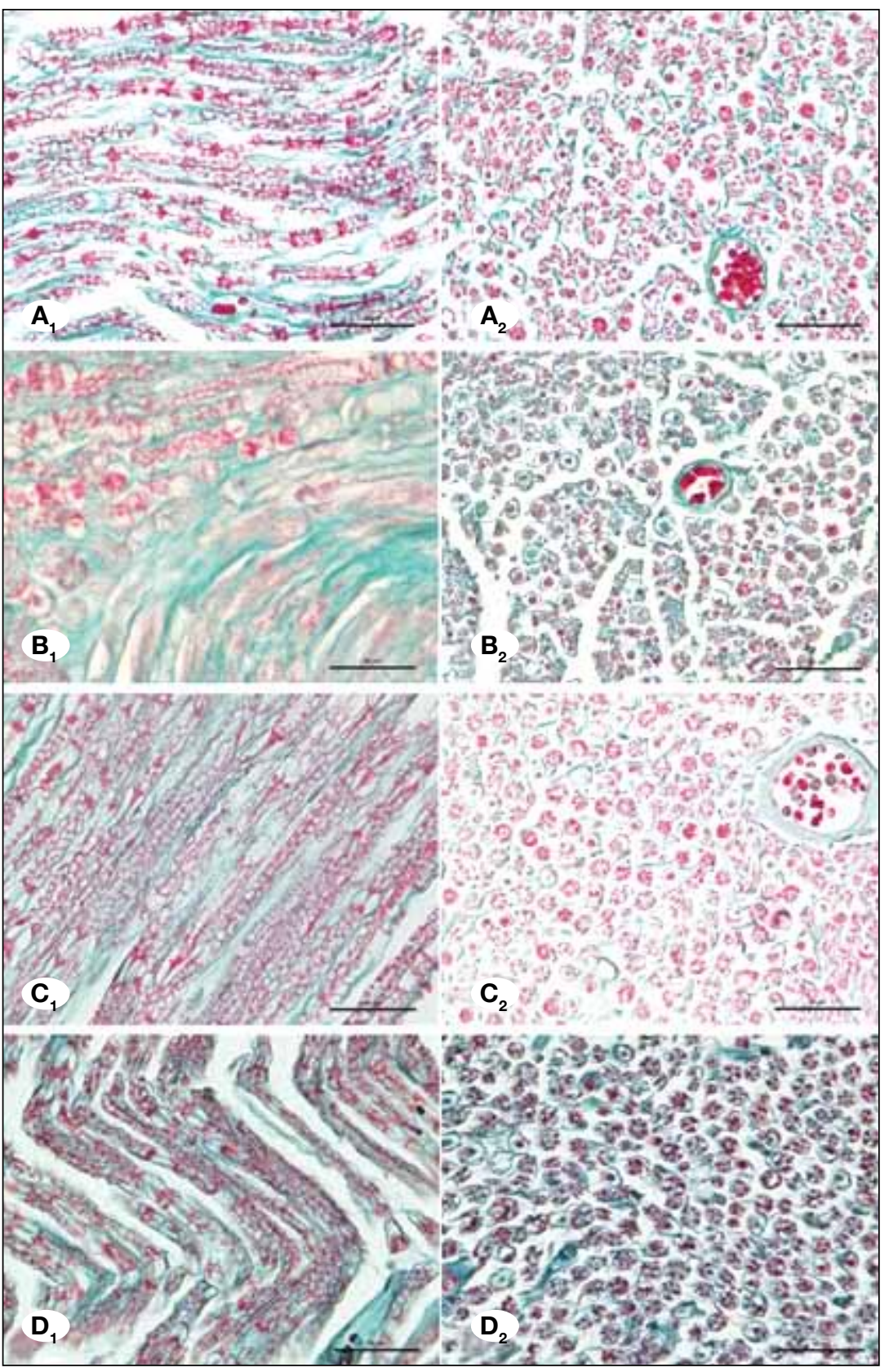

Figure 2: Control group showing normal morphology (A1-A2). Ischemia group showing axonal swelling and greatly high both axonal and myelin degeneration (B1-B2). Syringic acid group showing less fiber degeneration (C1-C2). MP group showing less fiber degeneration than syringic acid group (D1-D2). (Photomicrographs of longitudinal $(A 1, B 1, C 1, D 1)$ and transverse sections $(A 2, B 2, C 2, D 2)$ from the rat sciatic nerves (Gomori Trichrome, X400, scale bar $=50 \mu \mathrm{m}$ ). 
Tokmak M. et al: The Axon Protective Effects of Syringic Acid

Table II: The Scores of Caspase-3 Positive Cells According to Schmeichel et al. (22)

\begin{tabular}{ccccc}
\hline Rats & \multicolumn{5}{c}{ Scores of the groups for caspase-3 expression } \\
\hline & Control & Ischemia & Syringic acid & MP \\
\hline $\mathbf{1}$ & 0 & 2 & 1 & 0 \\
\hline $\mathbf{2}$ & 0 & 1 & 2 & 1 \\
\hline $\mathbf{3}$ & 0 & 2 & 1 & 1 \\
\hline $\mathbf{4}$ & 0 & 1 & 1 & 1 \\
\hline $\mathbf{5}$ & 0 & 2 & 1 & 1 \\
\hline $\mathbf{6}$ & 0 & 2 & 0 & 2 \\
\hline $\mathbf{7}$ & 0 & 3 & 1 & 1 \\
\hline $\mathbf{8}$ & 0 & 2 & 2 & 0 \\
\hline $\begin{array}{l}\text { Mean } \\
\text { value }\end{array}$ & 0 & 1.88 & 1.13 & 0.88 \\
\hline
\end{tabular}

Table III: The Activity of Superoxide Dismutase (SOD), Levels of Malondialdehyde (MDA) and Nuclear Respiratory Factor-1 (NRF1) of Rat Sciatic Nerve Tissues

\begin{tabular}{|c|c|c|c|}
\hline Groups & $\begin{array}{c}\text { NRF1 } \\
\begin{array}{c}\text { (n sg.ml } \\
\text { protein }\end{array}{ }^{-1} \text { ) } \\
\end{array}$ & $\begin{array}{c}\text { SOD } \\
(\text { U.ml-1.mg } \\
\left.\text { protein }^{-1}\right)\end{array}$ & $\begin{array}{c}\text { MDA } \\
\text { (nmole.ml } \text { (n) }^{-1} \mathrm{mg} \\
\left.\text { protein }^{-1}\right)\end{array}$ \\
\hline Control & $10.52 \pm 0.85^{a}$ & $7.38 \pm 0.66^{a}$ & $9.94 \pm 0.39^{c}$ \\
\hline I/R & $2.72 \pm 0.62^{d}$ & $3.18 \pm 0.37^{c}$ & $23.66 \pm 2.28^{a}$ \\
\hline $\mathrm{I} / \mathrm{R}+\mathrm{SA}$ & $7.85 \pm 1.07^{c}$ & $5.50 \pm 0.25^{b}$ & $13.95 \pm 1.99 \mathrm{~b}$ \\
\hline MP & $9.27 \pm 0.66^{b}$ & $5.84 \pm 0.47^{b}$ & $11.62 \pm 1.48^{c}$ \\
\hline
\end{tabular}

I/R: Ischemia/Reperfusion, SA: Syringic acid, MP: Methylprednisolone $30 \mathrm{mg} / \mathrm{kg}$.

Means in the same column by the different letter are significantly different to the One-way ANOVA-Tukey's test $(p<0.05)$. Data was expressed as mean $\pm S D$.

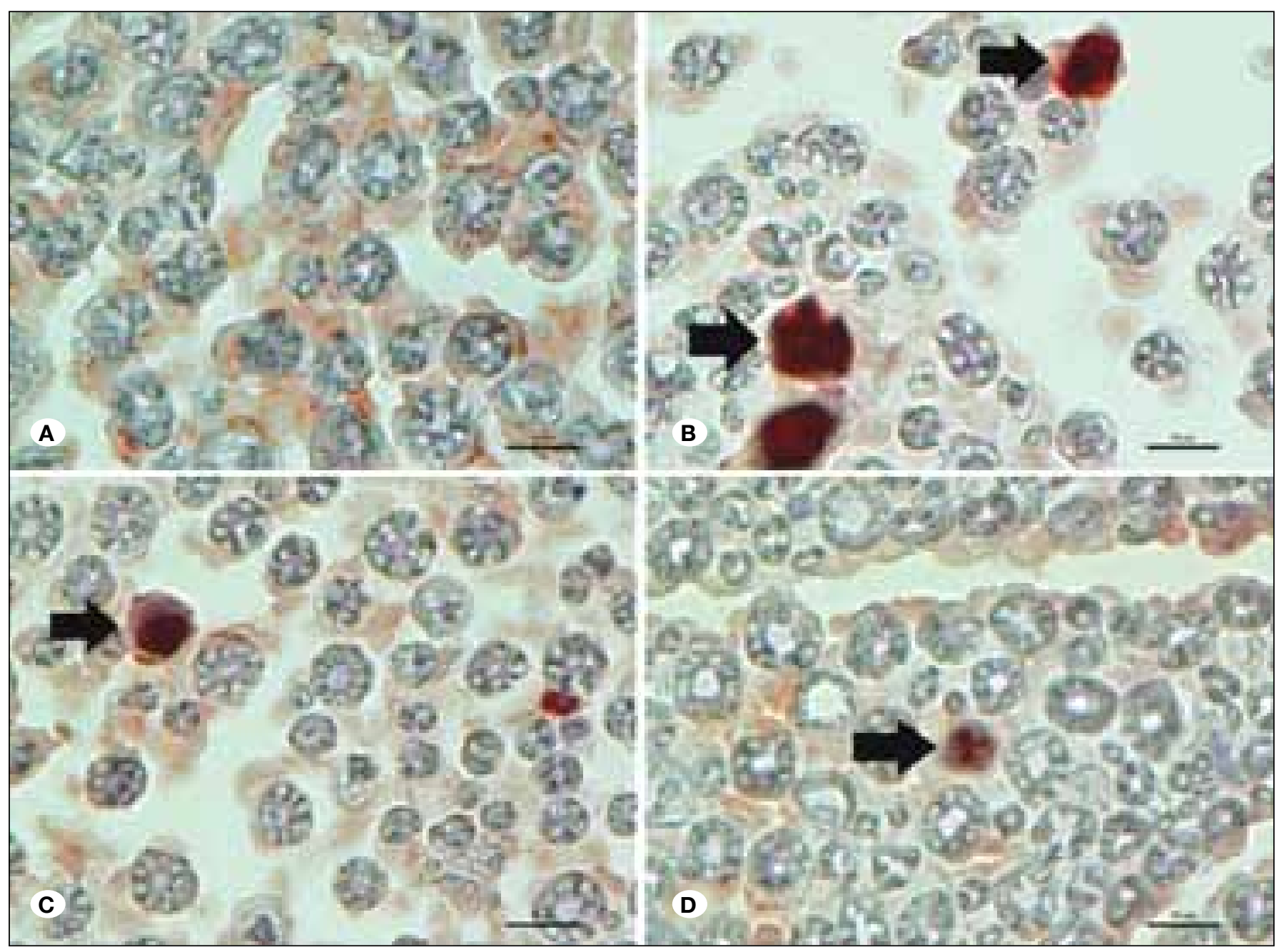

Figure 3: Control group showing no caspase-3 immunopositivity (A). Ischemia group showing increased number of caspase-3 positive fibers (B). Syringic acid group showing less caspase-3 immunopositivity than ischemia group (C). MP group showing less caspase-3 immunopositivity compared to ischemia (D). (Transverse sections of caspase-3 immunohistochemical staining from the sciatic nerves). (caspase-3 antibody, X1000, scale bar $=10 \mu \mathrm{m}$. Arrows indicate caspase-3 posivite nerve fibers). 


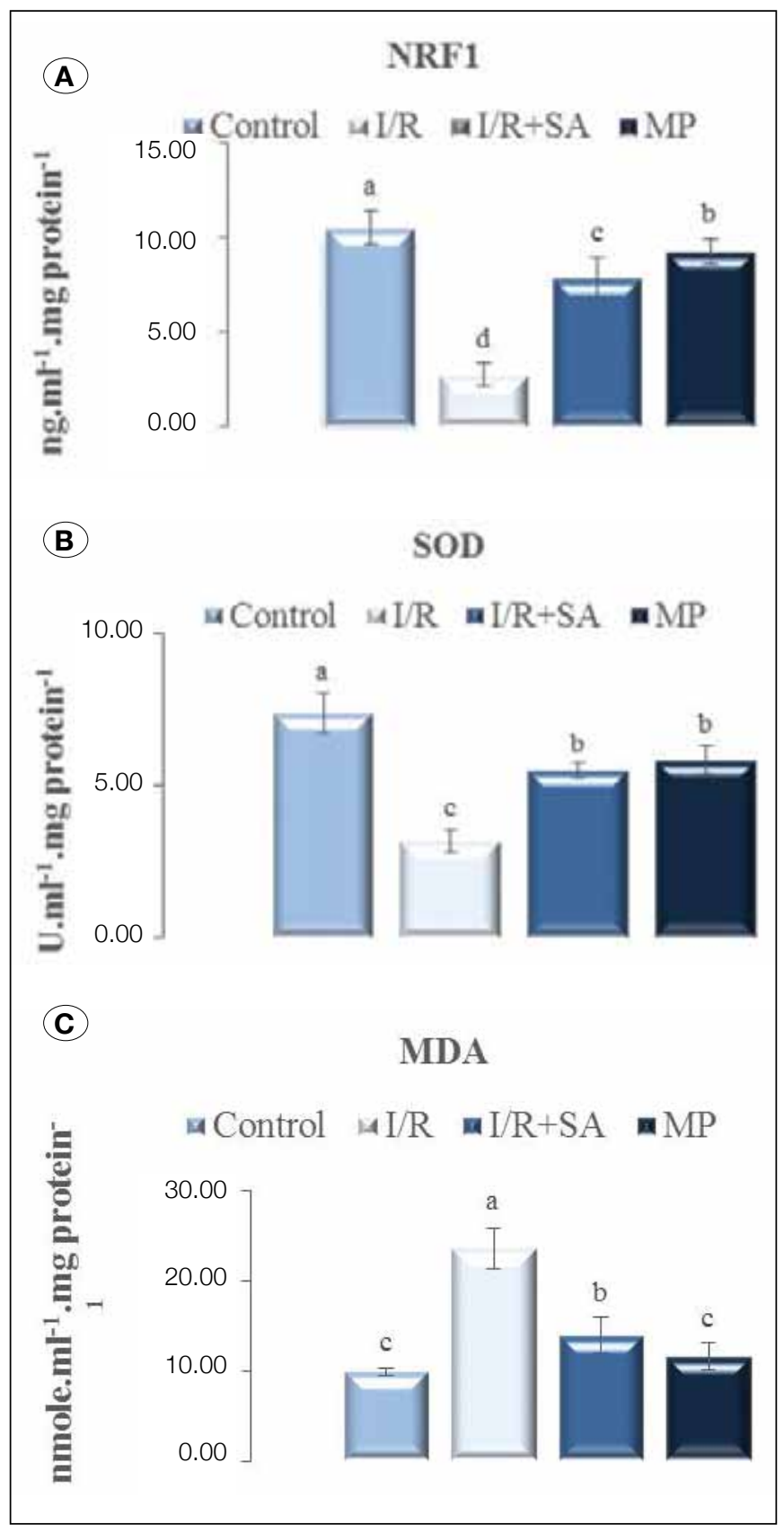

Figure 4: A) The effects of Syringic acid on changes in sciatic nerve tissue's nuclear respiratory factor-1 (NRF1) levels. ${ }^{b, c, d}$, $\mathrm{p}<0.05$ significantly different compared to the control group a. B) The effects of Syringic acid on changes in sciatic nerve tissue's enzyme activities of superoxide dismutase (SOD). Means in the same column by the same letter are not significantly different, b, $\mathrm{p}<0.05$ significantly different compared to control group ${ }^{\mathrm{a}}$ and ischemia/reperfusion group ${ }^{c}$. C) The effects of Syringic acid on changes in sciatic nerve tissue's malondialdehyde (MDA) levels. Syringic acid group is significantly different compared to other groups, $\mathrm{p}<0.05$. significant $(p<0.05)$. There was a significant difference between Syringic acid group and ischemia/reperfusion group $(p<0.05)$ like MP group. SOD activities of syringic acid group were significantly different compared to the control and ischemia/reperfusion groups $(p<0.05)$. Syringic acid group was not statistically different compared to MP group ( $p>0.05)$ but highly significant. MDA levels of ischemia/reperfusion group were found to be significantly higher than each group and syringic acid group was statistically significant compared to each other $(p<0.05)$.

\section{- DISCUSSION}

Although the mechanism for peripheral nerve injury after ischemia and reperfusion is poorly understood, various mechanisms have been proposed $(3,17,23,24)$. Axons are more resistant to ischemia than neurons since they have lower energy requirements $(12,24)$. Axonal ischemia and reperfusion leads to acute inflammatory changes including the influx of leukocytes at the lesion site in peripheral nerves thus inducing an inflammatory and metabolic cascade leading to cellular edema and disruption of cellular integrity $(3,8,17)$. In this study, we used a polyphenol that can be obtained naturally from plants known as SA. In the induced sciatic nerve ischemia model in rats, SA exerted a histopathologically verified axonoprotective effect on the ischemic sciatic nerve segments. Furthermore, following the treatment with SA, MDA level was decreased and SOD and NRF-1 values increased in biochemical investigations, indicating a significant reduction in the oxidative stress after ischemia/reperfusion. Since there is no definitive evidence regarding the toxicity of SA in human and animals, it is not considered to be toxic by ingestion and inhalation. However, it may cause skin irritation, allergic reactions, nausea and vomiting and exacerbate the ongoing liver and pulmonary diseases.

Surgical approaches to the descending aorta (cross-clamping the aorta) for thoraco-abdominal aortic diseases may result in temporary or permanent ischemia of the peripheral nerves including the sciatic nerve and its branches. Several strategies such as distal aortic perfusion, intrathecal vasodilators, reattachment of intercostal and lumbar vessels, and decreasing cerebrospinal fluid pressure have been used to overcome the hypoperfusion. Other measures including the free radical scavengers and immune system modulation also help to decrease the reperfusion injury $(1,10,19,21)$.

It is reported that polyphenol compounds exert anti-inflammatory and immunomodulatory effects via clearing ROS in oxidative stress and are commonly found naturally in herbs and herbal preparations containing biflavonoids. Although biflavonoids are known to display a variety of biological activities, such as anti-inflammatory activity, inhibition of cytochrome P450 enzymes, and antiviral activity, their neuroprotective roles have not been known $(7,9,16)$. Simonyi et al. demonstrated the neuroprotective effects of polyphenols in cerebral ischemia (23). Additionally, Morton et al. revelaed that SA helps in degrading free radicals by inhibiting the oxidation of low density lipoproteins as well as decelerates atherosclerosis by decreasing MDA production (18). Biflavonoids and particu- 
larly SA demonstrated a neuroprotective effect against ROSinduced insult $(10,21)$. Kang et al. reported that biflavonoids neuroprotection might be mediated by a direct blockade of the cell death cascades, but not by their anti-oxidative activity (11). To test this idea further, they examined the neuroprotective effects of biflavonoids against cytotoxic insult induced by staurosporine, which has been known to mediate apoptosis via the caspase- dependent mitochondrial pathway. They also suggested that biflavonoid neuroprotection appeared to be mediated, in part if not all, by direct blockade of the signaling events leading to apoptosis upon cellular stresses. However, in the current literature, an experimental study regarding the axon protective effects of SA on sciatic nerves does not exist.

We found that $S A$ as a biflavonoid appeared to have an axonoprotective effect on the sciatic nerves histopathologically. In addition to these histopathological finding, syringic acid decreased the MDA levels and increased the SOD and NRF-1 activities in biochemical analysis reducing oxidative stress after ischemia. Akdemir et al. reported that aortic clamping resulted in increase in MDA levels in peripheral nerves and edema that leads to axonal injury and myelin damage (2). The occurrence of IFD and edema indicates that long-term ischemia/reperfusion has a devastating impact on the peripheral neurons and that there is a direct link between paraplegia, one of the most common postoperative complications of aorta surgery, and the cross-clamping. Mitsui et al. proposed that the molecular mechanisms of nerve ischemia are similar to those of tissues like heart, gut and brain, but modified in terms of their threshold to ischemic and reperfusion damage (17). Ischemia would result in phospholipase activation and phospholipid breakdown, liberating arachidonic acid with its cascade producing the prostaglandins and leukotrienes. Oxygen free radicals would be generated during ischemia and especially during reperfusion. The formation of lipid hydroperoxides would inhibit prostacyclin synthetase $(6,15,25)$. The increased biosynthetic rate of thromboxane A2 coupled with the ischemia-related inhibition of prostacyclin production by endothelial cells would result in vasoconstriction, aggravating the ischemic insult (6). Eventually, this fatal cascade leads to formation of MDA as the end product of lipid peroxidation and at the same time is considered as one of the most sensitive markers of lipid peroxidation. Akdemir et al. (2) reported that aortic clamping resulted in increase in MDA levels in peripheral nerves and edema that leads to axonal injury and myelin damage. Syringic acid may enable neural tissues to become more resistant to lipid peroxidation, thereby activating antioxidant enzymes. In our study, MDA values were reduced significantly after SA treatment as compared to the untreated control group. Additionally, there was no significant difference in MDA values between the MP group and SA group. Peripheral nerves harbour Schwann cells with potentially enzymatic and non-enzymatic anti-oxidant capacity to protect peripheral nerves from the harmful effects of the free oxygen radicals. SOD activity protects the proteins from the metal-catalyzed reaction between $\mathrm{O}_{2}$ and $\mathrm{H}_{2} \mathrm{O}_{2}$. Oxidative stress developing after peripheral nerve ischemia/reperfusion causes an increase in SOD activity. However, in cases of oxidative stress that lasts for a longer time and exceeds the capacity of SOD,
SOD activity may reduce $(1,4,6,14,20)$. Similarly, we found that the SOD activity in the SA and MP group was significantly increased in comparison to that of the control group.

During the breakdown of free oxygen radicals, mitochondria are important regulators of the metabolic activity of neuronal cells. Mitochondrial biogenesis is activated in response to cellular oxidative stress via environmental stimuli and other many different signals. NRF-1 is a mitochondrial transcription factor that activates the majority of genes coding subunits of the respiratory complex. Kumari et al. documented the decreased level of NRF-1 at the induced neural ischemia and an in increase during reperfusion period in normal and hyperglycemic rats (13). Likewise, we found that the SA and MP groups exhibited higher NRF-1 values compared to the Sham group. Activation of caspase- 3 is known to be important and irreversible in apoptosis caused by ischemia of neuronal cells $(3,4,13)$. Recently, Unsain et al. reported that caspase-3 negative axons are protected from degeneration (26).

\section{- CONCLUSION}

In our study, SA and MP groups displayed fewer caspase3 immunopositive fibers than Control and Sham groups. We, for the first time, demonstrated the axon protective role of syringic acid in an animal model by histopathological analysis and biochemical means. Lack of neurophysiological evaluation of rats by electromyography was a limitation of our study. In conclusion, syringic acid may become an alternative or a supplementary agent to MP in the treatment of axonal ischemia/reperfusion injury.

\section{- REFERENCES}

1. Abas F, Alkan T, Goren B: Neuroprotective effects of postconditioning on lipid peroxidation and apoptosis after focal cerebral ischemia/reperfusion injury in rats. Turk Neurosurg 20:1-8, 2010

2. Akdemir O, Akdemir I, Cavusoglu T, Lineaweaver WC, Ates $U$, Zhang F, Erbas O: Impact of aortic cross-clamping time on peripheral nerves: Experimental model. Ann Thorac Cardiovasc Surg 21(1):72-77, 2015

3. Ban M, Tonai T, Kohno T, Matsumoto K, Horie T, Yamamoto S, Moskowitz MA, Levine L: A flavonoid inhibitor of 5-lipoxygenase inhibits leukotriene production following ischemia in gerbil brain. Stroke 20:248-252, 1989

4. Bone DB, Antic M, Quinonez D, Hammond JR: Hypoxanthine uptake by skeletal muscle microvascular endothelial cells from equilibrative nucleoside transporter 1 (ENT1)-null mice: Effect of oxidative stress. Microvasc Res 98:16-22, 2015

5. Bors W, Heller W, Michel C, Saran M: Flavonoids as antioxidants: Determination of radical-scavenging efficiencies. Methods Enzymol 186: 343-355, 1990

6. Buege JA, Aust SD: Microsomal lipid peroxidation. Methods Enzymol 52: 302-310, 1978

7. Cassidy A, Brown JE, Hawdon A, Faughnan MS, King LJ, Millward J, Zimmer-Nechemias L, Wolfe B, Setchell KD: Factors affecting the bioavailability of soy isoflavones in humans after ingestion of physiologically relevant levels from different soy foods. J Nutr 136: 45-51, 2006 
8. Grace PA: Ischaemia-reperfusion injury. Br J Surg 81:637647,1994

9. Hertog MGL, Hollman $\mathrm{PCH}$, van de Putte B: Content of potentially anticarcinogenic flavonoids of tea infusions, wines, and fruit juices. J Agric Food Chem 41:1242-1246, 1993

10. Hwang YW, Kim SY, Jee SH, Kim YN, Nam CM: Soy food consumption and risk of prostate cancer: A meta-analysis of observational studies. Nutr Cancer 61: 598-606, 2009

11. Kang SS, Lee JY, Choi YK, Song SS, Kim JS, Jeon SJ, Han $\mathrm{YN}$, Son KH, Han BH: Neuroprotective effects of naturally occurring biflavonoids. Bioorg Med Chem Lett 15:3588-3591, 2005

12. Kindy $M$ : Inhibition of tyrosine phosphorylation prevents delayed neuronal death following cerebral ischemia. J Cereb Blood Flow Metab 13:372-377, 1993

13. Kumari S, Anderson L, Farmer S, Mehta SL, Li PA: Hyperglycemia alters mitochondrial fission and fusion proteins in mice subjected to cerebral ischemia and reperfusion. Transl Stroke Res 3:296-304, 2012

14. Li P, Nijhawan D, Budihardjo I, Srinivasula SM, Ahmad M, Alnemri ES, Wang X: Cytochrome $C$ and dATP-dependent formation of Apaf-1/caspase-9 complex initiates an apoptotic protease cascade. Cell 91:479-489, 1997

15. Lowry $\mathrm{OH}$, Rosebrough NJ, Farr AL, Randall RJ: Protein measurement with the Folin phenol reagent. J Biol Chem 193: 265-275, 1951

16. Mathey J, Lamothe V, Coxam V, Potier M, Sauvant P, BennetauPelissero C: Concentrations of isoflavones in plasma and urine of post-menopausal women chronically ingesting high quantities of soy isoflavones. J Pharm Biomed Anal 41:957965, 2006

17. Mitsui Y, Schmelzer JD, Zollman PJ, Mitsui M, Kihara M, Low PA: Hypothermic neuroprotection of peripheral nerve of rats from ischemia-reperfusion injury: Intraischemic vs. reperfusion hypothermia. Brain Res 827(1-2):63-69, 1999
18. Morton LW, Croft KD, Puddey IB, Byrne L: Phenolic acids protect low density lipoproteins from peroxynitrite-mediated modification in vitro. Brain Res 827(1-2):63-69, 1999

19. Nizamutdinova IT, Oh HM, Min YN, Park SH, Lee MJ, Kim JS, Yean MH, Kang SS, Kim YS, Chang KC, Kim HJ: Paeonol suppresses intercellular adhesion molecule-1 expression in tumor necrosis factor-alpha-stimulated human umbilical vein endothelial cells by blocking p38, ERK and nuclear factorkappa B signaling pathways. Int Immunopharmacol 7:343350, 2007

20. Porter AG, Janicke RU: Emerging roles of caspase-3 in apoptosis. Cell Death Differ 6:99-104, 1999

21. Sakamoto T, Horiguchi H, Oguma E, Kayama F: Effects of diverse dietary phytoestrogens on cell growth, cell cycle and apoptosis in estrogen-receptor-positive breast cancer cells. J Nutr Biochem 21:856-864, 2010

22. Schmeichel AM, Schmelzer JD, Low PA: Oxidative injury and apoptosis of dorsal root ganglion neurons in chronic experimental diabetic neuropathy. Diabetes 52(1):165-171, 2003

23. Simonyi A, Wang Q, Miller RL, Yusof $M$, Shelat PB, Sun AY, Sun GY: Polyphenols in cerebral ischemia: Novel targets for neuroprotection. Mol Neurobiol 31(1-3):135-147, 2005

24. Stavric B: Quercetin in our diet: From potent mutagen to probable anticarcinogen. Clin Biochem 27:245-248, 1994

25. Sun Y, Oberley LW, Li Y: A simple method for clinical assay of superoxide dismutase. Clin Chem 34:497-500, 1988

26. Unsain N, Higgins JM, Parker KN, Johnstone AD, Barker PA: XIAP regulates caspase activity in degenerating axons. Cell Rep 4(4):751-763, 2013

27. Yuce S, Cemal Gökçe E, Işıkdemir A, Koç ER, Cemil DB, Gökçe A, Sargon MF: An experimental comparison of the effects of propolis, curcumin, and methylprednisolone on crush injuries of the sciatic nerve. Ann Plast Surg 74(6):684-692, 2015 